\title{
Envois de fonds des migrants, pauvreté et inégalités de revenu au Cameroun
}

Remittances, Poverty and Income Inequality in Cameroon

Las remesas de los emigrantes, la pobreza y la desigualdad de ingresos en

Camerún

Eric Stève Tamo Mbouyou

\section{(2) OpenEdition}

Journals

Édition électronique

URL : https://journals.openedition.org/remi/7001

DOI : $10.4000 /$ remi.7001

ISSN : $1777-5418$

Éditeur

Université de Poitiers

Édition imprimée

Date de publication : 1 décembre 2014

Pagination : $181-200$

ISBN : 979-10-90426-23-8

ISSN : 0765-0752

Référence électronique

Eric Stève Tamo Mbouyou, "Envois de fonds des migrants, pauvreté et inégalités de revenu au Cameroun », Revue européenne des migrations internationales [En ligne], vol. 30 - n³ et 4 | 2014, mis en ligne le 01 décembre 2017, consulté le 15 avril 2022. URL : http://journals.openedition.org/remi/7001 ; DOI : https://doi.org/10.4000/remi.7001 


\section{Envois de fonds des migrants, pauvreté et inégalités de revenu au Cameroun}

\section{Eric Stève Tamo Mbouyou ${ }^{1}$}

\section{Introduction}

La globalisation des échanges à travers le monde a donné à l'émigration internationale de nouveaux attraits parmi lesquels une portée globale, grâce notamment à l'élargissement du bassin migratoire avec l'irruption d'acteurs nouveaux et le développement de l'éventail de destinations. Sa complexification sans cesse croissante suscite plus que par le passé un renouvellement des questionnements qui lui sont liés, tant ses enjeux sont aussi importants que multiples : économiques, sociaux, culturels, politiques, sécuritaires, etc.

Cette émigration, quoiqu'elle pose de nombreux problèmes avec des conséquences non seulement dans les pays d'accueil, mais également dans les pays d'origine, s'accompagne d'une intensification des transferts matériels et immatériels, ce qui suscite désormais l'intérêt des décideurs politiques dans les pays d'émigration comme dans les pays d'arrivée. En effet, les envois de fonds ${ }^{2}$ des migrants vers leur pays d'origine, qui constituent la principale retombée de cette émigration, étaient en 2010 évalués à 440 milliards de dollars, dont 325 milliards (soit $74 \%$ ) à destination des pays en développement (Ratha, 2011).

Cet intérêt grandissant, sans cesse réaffirmé au plan international, s'est traduit par la tenue successive de plusieurs conférences internationales dans le cadre du Dialogue de haut niveau sur les migrations internationales et le développement, dont celui de 2013 a permis de souligner non seulement la contribution importante des migrations à la réalisation des Objectifs du Millénaire pour le Développement (OMD), mais également de reconnaitre la mobilité humaine comme un facteur décisif du développement durable ${ }^{3}$.

\footnotetext{
1 Chercheur associé à la Cellule d'Appui à la Recherche et à I'Enseignement des Institutions Francophones d'Afrique (CARE-IFA/UIESP), BP 660 Yaoundé, Cameroun ; tamoerics@gmail.com

2 Les termes " envois de fonds ", " transferts de fonds " et " transferts monétaires " sont indifféremment utilisés dans le cadre de cet article.

3 Projet de résolution de I'Assemblée générale des Nations Unies à l'occasion du Dialogue de haut niveau sur les migrations internationales et le développement, les 3 et 4 octobre 2013.
} 
Cependant, la question des transferts monétaires continue de faire l'objet de plusieurs controverses dans le débat relatif aux coûts et bénéfices de la migration internationale. D'un côté, on peut argumenter que les fonds transférés ont un impact positif tant en constituant une importante source de revenu additionnel pour de nombreux ménages (Zourkaléini et al., 2013), qu'en fournissant des devises fort utiles et nécessaires à l'importation de facteurs de production rares, non disponibles sur le plan intérieur (Ammassari, 2004). En revanche, et partant du fait que les fonds reçus sont généralement affectés aux besoins de consommation courante plutôt qu'à des fins productives, on peut opposer à cet argument celui selon lequel ceux-ci placent les bénéficiaires dans une situation de dépendance et provoquent, par ailleurs, une augmentation de la demande des biens d'importation au détriment des biens produits localement; quand bien même cette demande n'est pas tournée vers l'extérieur, il en résulte une pression inflationniste, ce qui à terme provoque une perte de compétitivité de I'économie (Gubert, 2005).

L'un des aspects les moins connus, notamment dans le cas de l'Afrique subsaharienne, est celui des conséquences de ces envois de fonds sur le bien-être des populations, notamment en ce qui concerne la pauvreté et les inégalités de revenu. II convient en effet de souligner que bien qu'étant, d'après la nouvelle économie de la migration, une stratégie familiale de couverture contre les imperfections du marché et l'incertitude (Stark, 1991), la migration internationale dans de nombreux cas concerne surtout les populations les mieux nanties, à même de supporter les coûts inhérents au projet migratoire. II en découle de ce fait que seule cette catégorie sociale en tire un réel profit particulièrement en termes de transferts (Adams et al., 2008 ; Adams, 1991 ; Barham et Boucher, 1998), ce qui nous conduit à nous interroger sur leur incidence véritable. Notre recherche a donc pour objectif d'évaluer la contribution des migrations internationales au développement des pays d'origine, notamment à travers la mesure de l'impact des envois de fonds sur la pauvreté et les inégalités de revenu au Cameroun. Afin de réaliser cet objectif, les données de l'enquête nationale sur l'impact des migrations Sud-Sud sur le développement au Cameroun réalisée en 2012 par I'Institut de Formation et de Recherche Démographiques (IFORD) avec le soutien de I'Organisation Internationale pour les Migrations (OIM) sont utilisées.

Ce travail s'articule autour de quatre points. Le premier présente les caractéristiques des transferts de fonds au Cameroun. Le deuxième donne un aperçu empirique de l'incidence des envois de fonds sur le bien-être des bénéficiaires. Le point suivant fixe le cadre méthodologique. Enfin, le dernier est une synthèse des résultats obtenus.

\section{Caractéristiques des transferts de fonds au Cameroun}

La crise économique qui a frappé le Cameroun tout comme la majorité des pays en développement au sud du Sahara dans les années 1980 fut à l'origine d'une montée de l'émigration économique aux cotés de celle d'étude, observée durant la période post indépendance. Dans ce contexte de crise caractérisé par un désengagement de l'État de ses fonctions régaliennes (éducation, santé, etc.), un chômage important, un manque de perspectives, une dégradation continue 
des conditions d'existence des populations, l'émigration constitua, aux niveaux individuel et familial, une stratégie de diversification des sources de revenus compte tenu de la situation d'incertitude ambiante, et les transferts de fonds constituèrent la principale passerelle reliant les migrants à leur pays d'origine (Kamdem, 2007 ; Guilmoto et Sandron, 2003).

Au cours de la décennie 2001-2010, les fonds transférés par les migrants à destination du Cameroun n'ont pratiquement pas cessé d'augmenter (Graphique 1). Cependant, la croissance quasi stable observée sur plusieurs années connait depuis 2008 une légère baisse consécutive à la crise financière mondiale, dont les effets se sont particulièrement fait ressentir dans les principaux pays d'émigration.

Graphique 1 : Évolution des transferts de fonds à destination du Cameroun (2001-2010)

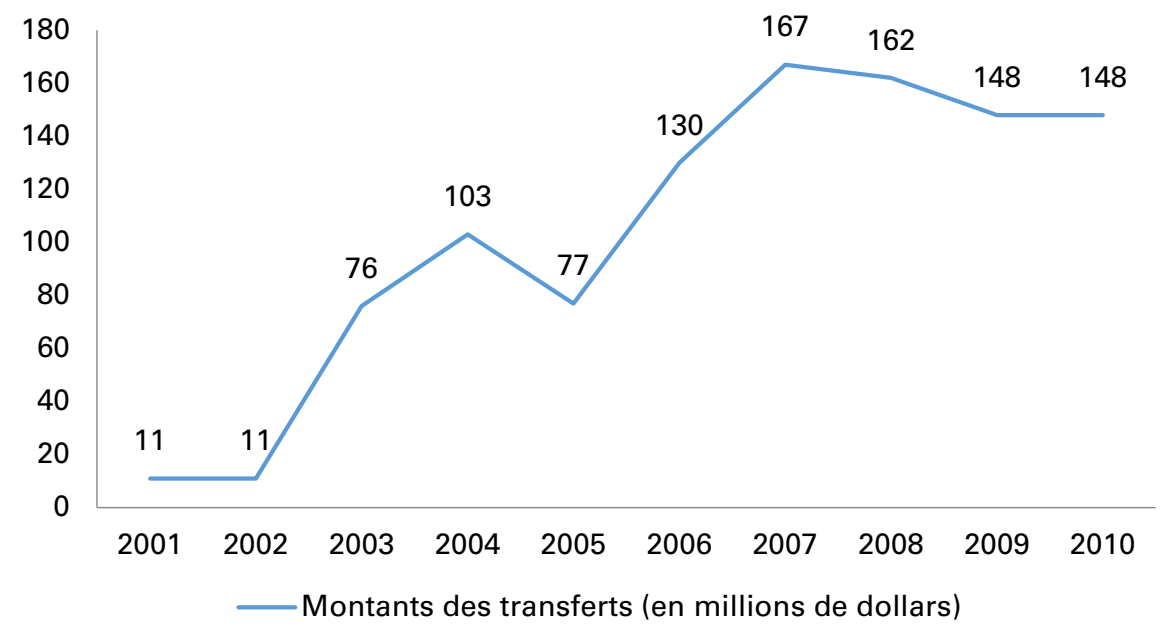

Source : Données du site www.worldbank.org/prospects/migrationandremittances, 2011.

Crédit : Eric Stève Tamo Mbouyou.

Évalués à 148 millions de dollars en 2010, ces fonds ne sont bien évidemment que l'une des conséquences des migrations. En 2000, la population d'émigrants camerounais était estimée à 170363 individus répartis majoritairement entre l'Afrique et l'Europe (DRC, 2007). La France, qui reste la première destination de ces émigrants, occupe également la première place devant les États-Unis en ce qui concerne l'entrée des fonds en provenance de l'étranger (Graphique 2). En 2010, les envois en provenance de ce pays s'élevaient à 54 millions de dollars, soit plus du tiers des fonds transférés au Cameroun cette même année. Ces deux pays sont suivis par I'Allemagne puis I'Italie pour ce qui relève des transferts Nord-Sud ; le Gabon, le Tchad et le Nigéria étant ces principaux pays d'envoi dans le cadre de transferts Sud-Sud. 
Graphique 2: Principaux pays d'origine des fonds transférés au Cameroun en 2010

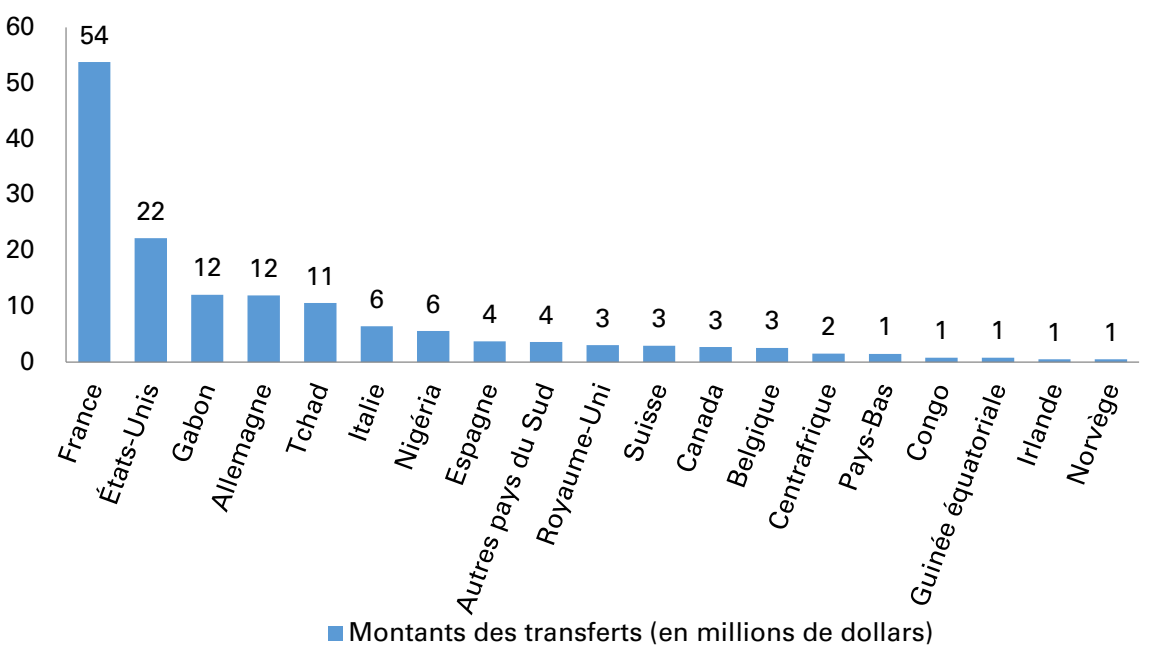

Source : Données du site www.worldbank.org/prospects/migrationandremittances, 2011. Crédit : Eric Stève Tamo Mbouyou.

L'altruisme, les arrangements familiaux tacites ou explicites et l'intérêt personnel constituent les motifs qui sous-tendent les transferts de fonds des migrants à destination du Cameroun. Leur caractère altruiste réside dans le fait qu'ils sont généralement destinés à l'entretien de la famille. Ils ont pour but d'aider à la satisfaction des frais de santé, de scolarisation, aux dépenses à caractère social et religieux ayant parfois une dimension ostentatoire (funérailles, mariages, baptêmes) à l'intérieur de la sphère familiale. Ils ont pour but d'aider celle-ci à satisfaire ses besoins courants : les principaux étant des cas de maladies et de décès survenus localement ainsi que la participation à la scolarisation des fratries et autres liens familiaux (Kamdem, 2007 ; Schmelz, 2007), car faut-il dire que le niveau de vie des populations en général reste encore assez faible. En 2007, 40 \% de la population totale du pays, soit environ 7,1 millions d'habitants, vivait en dessous du seuil de pauvreté fixé à 1,48 dollar par jour (INS, 2008).

En outre, les transferts de fonds constituent un aspect endogène du processus migratoire, dans la mesure où ils sont le fruit d'arrangements mutuellement bénéfiques : par exemple, lorsque le migrant rembourse les emprunts contractés par sa famille afin de financer le projet migratoire. Ils constituent également un indicateur de réussite personnelle facilitant une certaine ascension sociale du migrant. En effet, " dans le monde rural, il devient un notable local dont l'avis sur différentes questions est demandé par les membres de la famille et de la tribu, et il est fréquemment sollicité pour préparer le départ d'autres membres de la famille " (Khachani, 2007 : 213). En plus de titres " nobiliers" (Mimche, 2009), les opportunités d'investissements au plan économique constituent bien évidemment une source de motivation à l'origine de l'envoi de fonds par le migrant. 


\section{Impact des transferts de fonds sur le bien-être des ménages d'origine}

Le plus souvent présentées par de nombreuses formations politiques, principalement d'extrême droite, comme étant la principale responsable du malaise social observé en temps de " crise " dans les pays de destination, la migration et ses retombées sont en revanche perçues assez positivement dans les pays de départ. Au-delà de la simple mobilité sociale qu'elle confère fréquemment aux migrants, plusieurs acteurs de la sphère aussi bien politique qu'académique lui reconnaissent de plus en plus un rôle majeur dans le développement socioéconomique des pays d'origine (Afaha, 2013). Toutefois, l'impact de la migration sur le développement demeure assez difficile à cerner, étant donné la diversité des contextes et son caractère pluridimensionnel : impacts sur le développement humain, culturel et même politique (Adepoju, 2011).

Malgré une baisse de $5 \%$ en 2009, les transferts de fonds ont permis d'amortir les effets de la crise financière mondiale de 2007-2008. Cette baisse ne fut d'ailleurs que de très courte durée, car dès 2010 vint la reprise. Tel ne fut pas le cas des flux d'investissements directs étrangers (IDE) et des placements de portefeuille qui ont chuté respectivement de $40 \%$ et de $46 \%$ en 2009 (Ratha, 2011). Moins volatiles, les transferts de fonds sont peu sensibles aux variations cycliques de l'économie, ce qui permet de soutenir la consommation et les investissements lors de ralentissements économiques (Chami et al., 2008 ; Yang, 2008), grâce notamment, aux lissages des dépenses qu'ils autorisent. Au Cameroun, l'évolution à la hausse de la proportion des transferts de fonds par rapport au produit intérieur brut a, bien qu'étant faible, plutôt été stable entre 1979 et 2010 (Graphique 3), contrairement aux IDE et à I'aide publique au développement (APD) plus volatiles sous l'effet de la conjoncture. II convient cependant de préciser que l'absence de données fiables sur l'évolution des flux migratoires ne permet pas une confrontation entre la stabilité observée au niveau des transferts de fonds et les effectifs de migrants pour conclure à une réelle augmentation de ces derniers.

En l'absence d'un système de protection sociale et d'assurance, les transferts de fonds constituent une source de revenu additionnel importante pour de nombreuses familles. Ils permettent l'accès aux services sociaux de base comme l'éducation et la santé. À l'aide des données de l'enquête nationale sur le budget et la consommation des ménages au Niger, Boutin (2011) démontre que la réception de transferts internationaux par le ménage accroît la proportion d'enfants scolarisés d'environ $10 \%$. S'agissant de la santé, Franck et Hummer (2002) prouvent, dans le cas du Mexique, que la présence d'un émigrant au sein du ménage diminue le risque d'avoir un nouveau-né d'un poids inférieur à la normale. Les transferts de fonds contribuent par ailleurs à une baisse de la mortalité infantile (Lopez-Cordova, 2006). 
Graphique 3 : Évolution des flux d'IDE, APD et des transferts de fonds en direction du Cameroun (1979-2010)

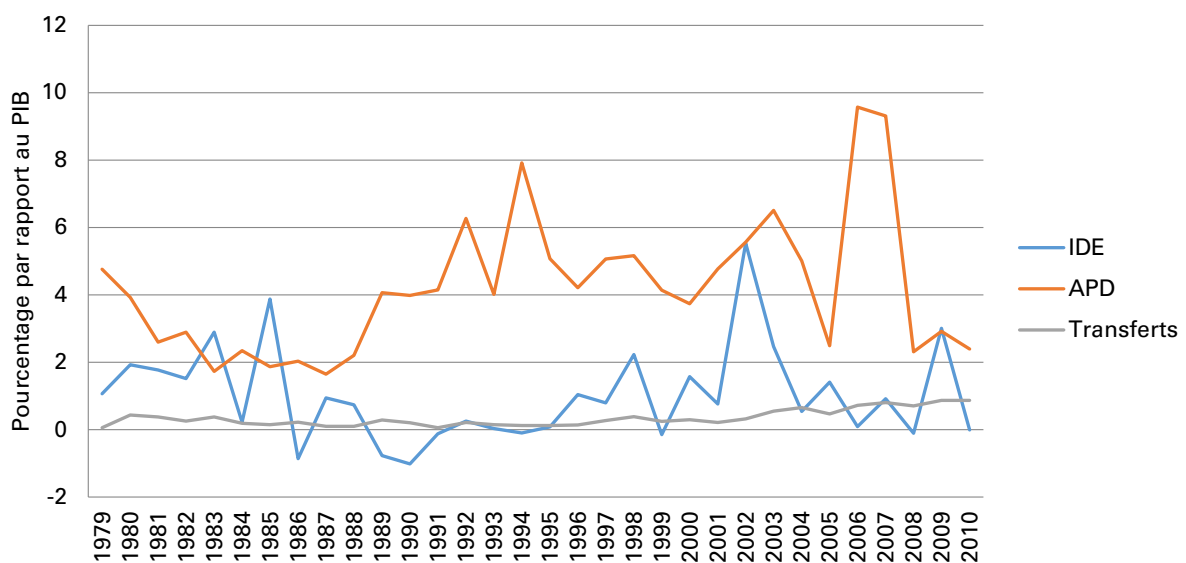

Source : Données du site http://donnees.banquemondiale.org, 2012.

Crédit : Eric Stève Tamo Mbouyou.

En ce qui concerne l'impact sur la pauvreté, Adams et Page (2005, 2003), Anyanwu et Erhijakpor (2010) démontrent à partir de données sur des pays en développement, parmi lesquels le Cameroun, qu'une hausse des transferts des migrants entraine une baisse du pourcentage d'individus vivant sous le seuil de pauvreté, soit un dollar par jour. Au Burkina Faso, les travaux de Lachaud sur ce sujet sont particulièrement illustratifs. Entre 1999 et 2005, ce pays a connu une hausse de la pauvreté du fait, selon l'auteur, de la baisse des transferts privés en provenance de Côte d'Ivoire, compte tenu de la crise militaro-politique qu'a traversée ce pays entre 1998 et 2003 (Lachaud, 1999 ; 2005). En considérant les envois de fonds comme des substituts potentiels des gains générés localement par le ménage, c'est-à-dire en l'absence de migration, Gubert et al. (2010) établissent que les transferts de fonds entrainent une réduction de la pauvreté au Mali. Utilisant le même scénario contrefactuel ${ }^{4}$ que précédemment, des résultats similaires ont été obtenus dans plusieurs pays d'Afrique, notamment au Ghana (Adams et al., 2008) et en Égypte (Adams, 1991).

Si l'impact positif des transferts de fonds sur la pauvreté semble être une évidence parmi les bénéficiaires, celui sur les inégalités de revenu est cependant plus difficile à établir. II n'est pas du tout possible d'affirmer avec certitude que les transferts de fonds permettent une convergence ou une divergence de revenu par rapport à la situation initiale, compte tenu de la diversité des contextes étudiés du point de vue des inégalités initiales, de celle des modèles migratoires et des disparités de résultats dues aux différences de méthode empirique appliquée (Wanner, 2008 ; Efionayi-Mäder et al., 2008 ; Gubert, 2005).

4 Le scénario " contrefactuel " est un terme fréquemment utilisé dans le cadre d'études similaires recourant à la même approche méthodologique, il permet de mesurer l'impact des transferts en partant du contrepied suivant : que gagnerait le ménage si, au lieu d'émigrer, le migrant restait et travaillait dans son pays d'origine? 
Si I'on peut observer au Mali (Gubert et al., 2010) de même qu'au Mexique (Taylor et al., 2005) une baisse de ces inégalités en présence des transferts internationaux, ceci n'est pas le cas du Ghana (Adams et al., 2008), ni de l'Égypte (Adams, 1991) et encore moins du Nicaragua (Barham et Boucher, 1998) où elles se renforcent et sont au cœur des dysfonctionnements sociaux. La principale explication tient ici au fait que les familles les mieux nanties, contrairement aux plus démunies, participent davantage à l'émigration internationale, car plus à même de supporter les coûts y afférents et bénéficient donc en retour plus largement de ses retombées.

\section{Aspects méthodologiques}

Après avoir présenté la source de données puis défini les principaux concepts utilisés, nous exposerons ensuite la méthode d'analyse employée et décrirons enfin les variables mobilisées dans le cadre de cette étude.

\section{Source de données}

Nos travaux mobilisent les données de l'enquête sur "L'impact des migrations Sud-Sud sur le développement au Cameroun " réalisée en 2012 par IFORD avec I'appui financier de I'OIM. L'enquête a porté sur 1235 ménages répartis sur l'ensemble du territoire national. Douze régions ont été choisies pour cette enquête. II s'agit des dix régions administratives du pays (Adamaoua, Centre, Est, Extrême-nord, Littoral, Nord, Nord-ouest, Ouest, Sud, Sud-ouest) auxquelles ont été ajoutées les villes de Yaoundé et Douala ; les régions du Centre et du Littoral ont donc été considérées respectivement sans ces deux villes.

Le plan de sondage appliqué fut de type aléatoire stratifié à deux degrés. Au premier degré, des départements ou unités primaires de sondage ont été sélectionnés à partir des poids d'émigration et d'immigration dans leur région d'appartenance et selon le milieu de résidence. Les départements tirés ont servi ensuite de base de sondage pour le tirage de quatre-vingt-deux villages/ quartiers (soixante et onze urbains et onze ruraux). Au second degré, un échantillon de ménages (quinze) dans chaque village/quartier (dont de préférence dix avec au moins un migrant et cinq sans migrant) a été tiré. Des coefficients de pondération ont été calculés et ajoutés aux fichiers de données ; ceux du fichier ménage étant l'inverse de la probabilité de sélection du ménage, calculée au niveau du domaine d'échantillonnage (région). Les pondérations des ménages ont été ajustées afin de tenir compte des non-réponses au niveau de chaque domaine, et ont été normalisées par un facteur constant de façon à ce que le nombre total extrapolé de ménages soit égal au nombre total de ménages nonpondéré.

La mesure des transferts de fonds portait sur une période donnée : les douze mois précédant l'enquête et concernait les ménages ayant connu le départ pour l'étranger d'au moins un de leurs anciens membres au cours de la période comprise entre août 2002 et la date de l'enquête. Au final, l'échantillon sera constitué de 659 ménages ne bénéficiant pas de transferts et 260 bénéficiaires. 


\section{Définition des concepts}

- Les transferts de fonds sont des échanges interindividuels mettant en relation deux entités géographiques, en l'occurrence deux pays. Ils désignent les biens en numéraire envoyés par le migrant à destination de son ménage d'origine. Dans le cadre de cette étude, seuls les transferts en provenance d'un ancien membre du ménage sont pris en compte étant donné le contrefactuel utilisé au plan méthodologique pour la mesure de leur impact sur le bien-être du ménage.

- Le ménage est un ensemble constitué par une ou plusieurs personnes apparentées ou non qui reconnaissent l'autorité d'une seule et même personne (le chef de ménage), vivent dans un même logement, prennent souvent leur repas en commun et subviennent en commun aux dépenses courantes. Les ménages bénéficiaires désignent ceux avec au moins un émigrant (entendu comme étant un ancien membre du ménage parti à l'étranger c'est-à-dire hors du Cameroun au cours des dix dernières années précédant l'enquête) et qui ont reçu de ce dernier au moins un transfert monétaire au cours des douze derniers mois précédant l'enquête. Les ménages non bénéficiaires représentent ceux sans un émigrant, et qui, par ailleurs, n'ont pas reçu d'une tierce personne (parents, amis, etc.) résidant hors du Cameroun le moindre transfert monétaire au cours des douze derniers mois précédant l'enquête.

- Pour mesurer la pauvreté, nous recourons dans cet article à l'approche monétaire. Celle-ci présente l'avantage de refléter la photographie de la société à un moment précis donné. En effet, les conditions d'existence utilisées dans l'approche non monétaire s'améliorent moins vite dans le temps, voire quasiment plus au-delà d'un certain standing, et sont par conséquent difficilement rattachables à l'émigration. Au Cameroun, les biens possédés par le ménage, de même que les caractéristiques de son habitat, ont de fortes chances d'être identiques avant, mais aussi après le départ du migrant, dans la mesure où les fonds reçus de ce dernier sont prioritairement destinés à la satisfaction des besoins de consommation courants plutôt qu'à l'immobilier, comme illustre le graphique 4.

L'approche monétaire évaluerait ainsi mieux le niveau de bien-être du ménage, car, tout comme les transferts fonds, sa mesure porte sur les douze derniers mois précédant l'enquête. Ce choix s'appuie également sur les travaux de plusieurs auteurs parmi lesquels Adams (1991) qui propose, pour évaluer l'impact des transferts, de confronter les parts de revenus que consacrent les ménages aux différentes dépenses en fonction de leur position sur l'échelle de revenus, mais aussi de leur statut migratoire.

La mesure de l'impact des transferts de fonds des migrants porte sur les indicateurs suivants : (i) la dépense par tête, (ii) l'incidence, la profondeur et la sévérité de la pauvreté et (iii) l'indice de Gini. 


\section{Graphique 4 : Principaux usages faits des fonds reçus par les ménages bénéficiaires}

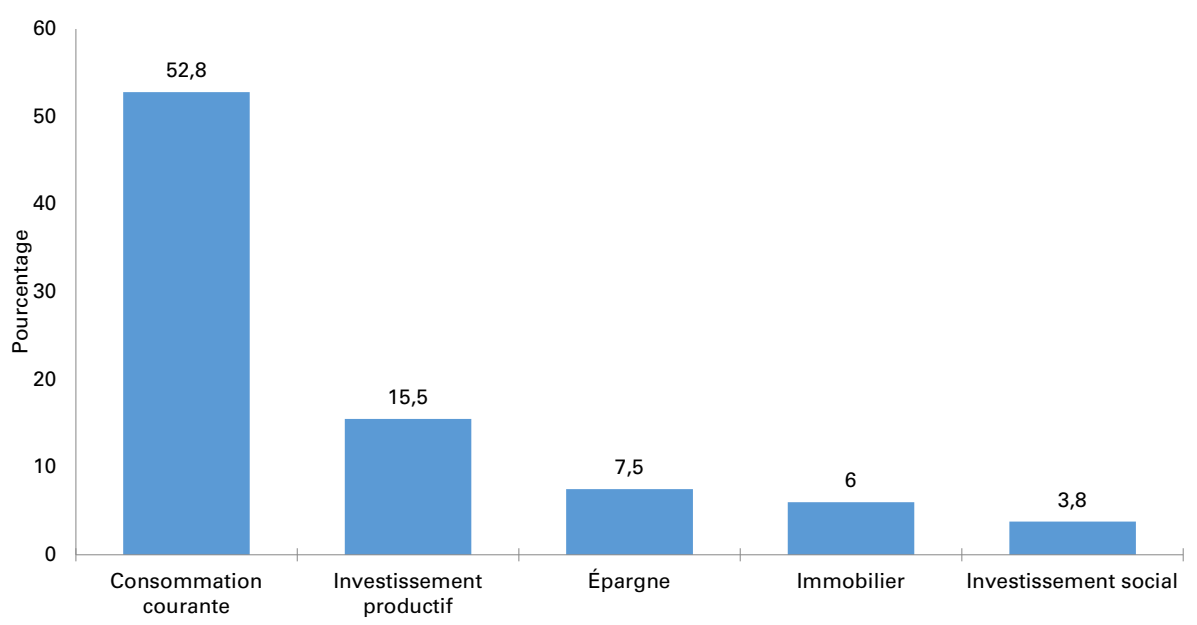

Source : Données de l'IFORD, 2012.

Crédit : Eric Stève Tamo Mbouyou.

Indicateur de bien-être monétaire, la dépense par tête (proxy du revenu par tête) désigne la dépense annuelle qu'effectue en moyenne un ménage pour chacun de ses membres. Elle prend en compte tous les besoins aussi bien biologiques (nutrition) que sociaux (santé, éducation, logement, habillement, loisir et transport) pouvant faire l'objet d'une valorisation monétaire. En ce qui concerne I'incidence de la pauvreté, elle se définit comme la proportion d'individus vivant sous le seuil de pauvreté. Ce seuil est fixé à 269443 francs CFA ${ }^{5}$ soit environ 539 dollars par personne et par an. S'agissant de la profondeur de la pauvreté, elle mesure la distance moyenne qui sépare les pauvres du seuil de pauvreté. La sévérité de la pauvreté mesure, quant à elle, la différence d'intensité (inégalité) de la pauvreté parmi les pauvres. Pour ce qui est de l'indice de Gini, il est utilisé pour la mesure des inégalités de revenu et est compris entre 0 et 1 . Plus il est proche de 1, plus l'inégalité mesurée est importante.

Ces trois indicateurs se calculent à l'aide de la formule suivante :

$$
P_{\alpha}=\frac{1}{n} \sum_{i=1}^{n}\left(\frac{Z-Y_{i}}{Z}\right)^{\alpha} \varepsilon
$$

- $Y_{i}$ représente la dépense par tête du ménage i et $Z$ le seuil de pauvreté ;

- $\varepsilon$ est une fonction indicatrice qui prend la valeur 1 si $Z>Y_{i}$ et 0 sinon.

Pour :

- $\alpha=0, P_{0}$ désigne l'incidence de la pauvreté ;

$-\alpha=1, \mathrm{P}_{1}$ désigne la profondeur de la pauvreté ;

$-\alpha=2, P_{2}$ désigne la sévérité de la pauvreté. 


\section{Méthodes d'analyse}

Afin de savoir si les transferts de fonds soulagent ou non la pauvreté, il est indispensable de suivre les trajectoires économiques, mais aussi migratoires des bénéficiaires, ou du moins de disposer d'une séquence d'observations. Or les panels de ménages perdent justement ceux qui migrent et les enquêtes biographiques rétrospectives, qui restent rares et lourdes, peinent à obtenir une appréciation objective des pauvretés passées (Delaunay, 2006).

Compte tenu donc du caractère transversal de nos données, nous comparons la situation observée, c'est-à-dire qui tient compte des transferts, à un scénario contrefactuel où le migrant resterait et travaillerait dans son pays d'origine. À l'aide de la méthode des Moindres Carrés Ordinaires (MCO), il s'agit précisément d'estimer le revenu des ménages non bénéficiaires et d'utiliser les coefficients estimés pour prédire celui des ménages bénéficiaires en absence de migration. La série ainsi obtenue est comparée aux revenus observés et l'écart qui en résulte est considéré comme la contribution des transferts de fonds au bien-être du ménage.

Cependant, une telle approche sous-tend implicitement que les fonds reçus par le ménage ont un impact sur son revenu actuel. Or un tel impact peut résulter des effets directs et/ou indirects des transferts antérieurs sur d'autres sources de revenus : d'où sa principale limite. Suivant cette approche, le revenu contrefactuel des ménages bénéficiaires s'obtient à partir de l'équation 1 suivante :

$$
\log Y_{i}=\alpha+\beta \log H_{i}+\gamma X_{i}+\varepsilon_{i}
$$

Où $Y_{i}$ est une mesure du revenu du ménage bénéficiaire $i . H_{i}$ et $X_{i}$ sont respectivement les vecteurs des caractéristiques du ménage et celles du chef de ménage, et $\varepsilon_{\mathrm{i}}$ est le terme d'erreur.

La prise en compte de la sélection endogène des ménages migrants, c'està-dire qui participe à l'émigration internationale, est indispensable pour éviter une invalidation de la méthode des MCO. En effet, les ménages migrants sont en général les plus dynamiques. Une estimation de leur revenu par la méthode des MCO serait ainsi sous-évaluée, car ces ménages auront vraisemblablement, du fait de leurs caractéristiques non observables, un revenu supérieur à celui des autres ménages même en absence de migration. Ce qui conduirait à une surévaluation de l'impact des transferts de fonds. Pour corriger ce biais endogénéité introduit par la sélectivité des ménages migrants, le mécanisme de correction utilisé ici s'inspire de celui de Barham et Boucher (1988). La règle de (non) sélection est modélisée à partir de la spécification probit suivante (équation 2) :

$$
\mathbf{M}_{\mathbf{i}}=\alpha_{\mathrm{m}}+\boldsymbol{\beta}_{\mathbf{m}} \log \mathbf{H}_{\mathbf{i}}+\gamma \mathbf{X}_{\mathbf{i}}+\delta_{\mathbf{m}} \mathbf{Z}_{\mathbf{i}}+\varepsilon_{\mathbf{i}}
$$

Où $M$ vaut 1 quand le ménage ne participe pas à l'émigration internationale et 0 sinon.

$Z_{i}$ est le vecteur de variables explicatives d'une non-participation du ménage à l'émigration internationale, mais sans effet sur le revenu de ménage ainsi que les caractéristiques non observables contenues dans $\varepsilon_{i}$. On fait l'hypothèse qu'un ménage migrant est doté d'une fonction de production générant un revenu $Y_{1}$; 
dans le cas contraire, sa fonction de production a des coefficients différents et génère un revenu $Y_{0}$.

À partir de l'équation 2, nous estimons la probabilité pour un ménage de ne pas participer à l'émigration internationale ; probabilité à partir de laquelle on construit l'inverse du ratio de Mill $(\lambda)$ qui permet de corriger les biais liés à la sélection endogène des ménages migrants. L'équation 1 peut ainsi de nouveau s'écrire (équation 3) :

$$
\log Y_{i}=\alpha+\beta \log H_{i}+\gamma X_{i}+\lambda_{i}+\mu_{i}
$$

Où $\mu_{i}$ est le nouveau terme d'erreur, indépendamment et identiquement distribué.

\section{Description des variables}

Les termes $H_{i}$ et $X_{i}$ des équations 1, 2 et 3 sont respectivement les vecteurs des caractéristiques du ménage et celles du chef de ménage. Il s'agit précisément, au niveau du ménage : du lieu de résidence, d'actifs possédés, de la taille et du nombre d'individus instruits (ayant un niveau secondaire ou plus) qu'il abrite. Notons que la variable " actifs possédés " est un score de biens détenus par le ménage, construit à l'aide d'une analyse en composante principale (ACP) à partir des biens suivants : téléphone, ordinateur, foyer amélioré, machine à coudre, bicyclette, moto, voiture, porte-tout, outils de production agricole, électricité et eau. Ces actifs forment avec le capital humain, opérationnalisé à travers le nombre d'individus instruits, les facteurs qui déterminent le niveau de production du ménage et par conséquent son revenu. Les caractéristiques du chef de ménage sont, quant à elles, appréhendées à travers l'âge, le sexe et la situation d'activité de celui-ci.

Le vecteur $Z_{i}$ de variables explicatives d'une non-participation du ménage à l'émigration internationale - contenu dans l'équation de (non) sélection ou équation 2 - est saisi par la seule variable "langue parlée par le ménage ${ }^{6}$. En effet, l'émigration au Cameroun se caractérise par une forte préférence pour les pays ayant un fond linguistique commun : les migrants s'exprimant en français ont une préférence pour des pays tels que la France, le Gabon et leTchad, tandis que ceux d'expression anglaise choisissent les États-Unis et le Nigéria comme lieux de destination (Tableau 1). Dès lors, la propension du ménage à participer ou non à l'émigration internationale varie selon la langue dans laquelle il s'exprime (cf. Tableau 6 en annexe).

6 II s'agit des langues officielles que sont le français et I'anglais. Le choix des variables, ici, est loin d'être un exercice facile, car celles-ci doivent, tout en n'ayant aucun effet sur le revenu du ménage ainsi que les caractéristiques non observables contenues dans le terme d'erreur de l'équation de revenu, influer sur la propension du ménage à participer à l'émigration internationale. La solution couramment utilisée consiste à recourir à des mesures de composition ethnique ou de concentration géographique, dans la mesure où la migration est souvent un phénomène lié à des réseaux et à des pratiques ethniques (Gubert et al., 2010 ; Adams et al., 2008 ; Lachaud, 1999). 
Tableau 1 : Langue officielle camerounaise parlée par le migrant selon le pays de destination

\begin{tabular}{|l|l|l|l|l|}
\hline \multirow{2}{*}{\begin{tabular}{c}
\multirow{2}{*}{$\begin{array}{c}\text { Pays de } \\
\text { destination }\end{array}$} \\
\cline { 2 - 4 }
\end{tabular}} & \multicolumn{1}{|c|}{ Langue officielle parlée par le migrant } & \multirow{2}{*}{ Ensemble } \\
\hline France & 79,38 & \multicolumn{1}{|c|}{ Anglais } & \multicolumn{1}{c|}{ Bilingue } & \\
\hline États-Unis & 21,74 & 4,12 & 16,49 & 100 \\
\hline Gabon & 76,81 & 50,00 & 28,26 & 100 \\
\hline Tchad & 65,38 & 4,35 & 18,84 & 100 \\
\hline Nigéria & 21,82 & 0,00 & 34,62 & 100 \\
\hline Allemagne & 51,61 & 23,64 & 54,55 & 100 \\
\hline Italie & 66,67 & 32,26 & 16,13 & 100 \\
\hline
\end{tabular}

Source : Données de l'IFORD, 2012.

Crédit : Eric Stève Tamo Mbouyou.

Tableau 2 : Description des ménages bénéficiaires et non récipiendaires de transferts

\begin{tabular}{|c|c|c|c|c|c|c|}
\hline \multirow[t]{2}{*}{ Variables } & \multicolumn{2}{|c|}{$\begin{array}{c}\text { Ménages non } \\
\text { bénéficiaires } \\
(n=659)\end{array}$} & \multicolumn{2}{|c|}{$\begin{array}{c}\text { Ménages } \\
\text { bénéficiaires } \\
(n=260)\end{array}$} & \multicolumn{2}{|c|}{$\begin{array}{c}\text { Ensemble } \\
(\mathrm{n}=919)\end{array}$} \\
\hline & Moyenne & Écart-type & Moyenne & Écart-type & Moyenne & Écart-type \\
\hline Dépense par tête & 2512677 & 8767578 & 1779036 & 7259866 & 2305793 & 8372606 \\
\hline $\begin{array}{l}\text { Ménage résidant à } \\
\text { Douala/Yaoundé* }\end{array}$ & 0,27 & 0,45 & 0,31 & 0,46 & 0,28 & 0,45 \\
\hline $\begin{array}{l}\text { Ménage résidant dans } \\
\text { une autre ville urbaine* }\end{array}$ & 0,50 & 0,50 & 0,47 & 0,50 & 0,49 & 0,50 \\
\hline $\begin{array}{l}\text { Ménage résidant en } \\
\text { milieu rural* }\end{array}$ & 0,23 & 0,42 & 0,22 & 0,41 & 0,23 & 0,42 \\
\hline $\begin{array}{l}\text { Score d'actifs possédés } \\
\text { par le ménage }\end{array}$ & $-0,30$ & 1,39 & 0,31 & 1,28 & $-0,13$ & 1,38 \\
\hline Taille du ménage & 4,66 & 2,65 & 4,78 & 2,49 & 4,70 & 2,61 \\
\hline $\begin{array}{l}\text { Nombre d'instruits } \\
\text { (secondaire ou plus) }\end{array}$ & 1,81 & 1,76 & 2,63 & 2,06 & 2,04 & 1,88 \\
\hline $\begin{array}{l}\text { Femme chef de } \\
\text { ménage* }^{*}\end{array}$ & 0,18 & 0,38 & 0,35 & 0,48 & 0,23 & 0,42 \\
\hline Âge du chef de ménage & 41,86 & 13,76 & 49,51 & 15,63 & 44,02 & 14,71 \\
\hline $\begin{array}{l}\text { Âge du chef de ménage } \\
\text { au carré }\end{array}$ & 1941,02 & 1305,60 & 2694,42 & 1631,76 & 2153,70 & 1444,85 \\
\hline $\begin{array}{l}\text { Chef de ménage non } \\
\text { occupé* }\end{array}$ & 0,24 & 0,43 & 0,40 & 0,49 & 0,29 & 0,45 \\
\hline $\begin{array}{l}\text { Langue parlée : français } \\
\text { uniquement* }\end{array}$ & 0,58 & 0,49 & 0,54 & 0,50 & 0,57 & 0,50 \\
\hline $\begin{array}{l}\text { Langue parlée : anglais } \\
\text { uniquement* }\end{array}$ & 0,10 & 0,30 & 0,19 & 0,39 & 0,13 & 0,33 \\
\hline $\begin{array}{l}\text { Langue parlée : français } \\
\text { et anglais* }\end{array}$ & 0,32 & 0,47 & 0,27 & 0,45 & 0,30 & 0,46 \\
\hline
\end{tabular}

* Variable muette.

Source : Données de I'IFORD, 2012.

Crédit : Eric Stève Tamo Mbouyou. 
D'après le tableau 2, les ménages bénéficiaires de transferts de fonds résident principalement en zone urbaine et ont un score d'actifs supérieur à celui des ménages non récipiendaires. En outre, les ménages qui en dépendent le plus sont ceux dirigés par des femmes, mais également ceux ayant à leur tête un chef n'exerçant aucune activité économique.

\section{Principaux résultats}

L'estimation du revenu des ménages non bénéficiaires nous permet d'obtenir les coefficients servant à prédire celui des ménages récipiendaires en l'absence de migration. Dans l'ensemble, les signes de ces coefficients sont conformes aux attentes théoriques (Tableau 3).

Tableau 3 : Estimation du revenu des ménages sans migrant

\begin{tabular}{|c|c|c|}
\hline \multirow{2}{*}{ Variables } & $\begin{array}{c}\text { Sans } \\
\text { correction }\end{array}$ & $\begin{array}{c}\text { Avec } \\
\text { correction }\end{array}$ \\
\hline & \multicolumn{2}{|c|}{ Coefficients } \\
\hline Constante & $14,0872 * * *$ & $14,0872 * * *$ \\
\hline \multicolumn{3}{|l|}{ Milieu de résidence } \\
\hline Yaoundé/Douala & Réf. & Réf. \\
\hline Urbain & $-1,2875^{* * *}$ & $-1,3146 * * *$ \\
\hline Rural & $-1,6777 * * *$ & $-1,7080 * * *$ \\
\hline Score d'actifs possédés (Log) & $0,2366^{* *}$ & 0,0665 \\
\hline Taille du ménage (Log) & $-0,6501 * * *$ & $-0,5539 * * *$ \\
\hline Nombre d'instruits (Log) & $0,2462 * *$ & 0,0504 \\
\hline Sexe du chef de ménage $(0=$ Homme, $1=$ Femme $)$ & $-0,4288 * * *$ & $-0,6028 * * *$ \\
\hline Âge du chef de ménage & 0,0074 & 0,0068 \\
\hline Âge du chef de ménage au carré & $-0,0002$ & $-0,0003$ \\
\hline Situation d'activité du CM (0 = Non occupé, 1 = Occupé) & $0,2368^{*}$ & $0,2930 * *$ \\
\hline Inverse du ratio de Mill (Lambda) & NA & $1,3619 * *$ \\
\hline $\mathrm{R}^{2}=$ & $38,4 \%$ & $40 \%$ \\
\hline
\end{tabular}

Variable dépendante : Log (dépenses par tête) avec Log = logarithme.

$$
\begin{aligned}
& \text { NA : non applicable. } \\
& *^{* *} p<0,001 \text {; }{ }^{* *} p<0,05 \text {. }
\end{aligned}
$$

Source : Données de l'IFORD, 2012.

Crédit : Eric Stève Tamo Mbouyou.

Le revenu du ménage varie en fonction du lieu de résidence. Les ménages résidant dans les villes de Yaoundé et Douala ont un revenu supérieur à celui des ménages se localisant dans les autres villes urbaines du pays et plus encore à celui des ménages ruraux, toutes choses étant égales par ailleurs. D'autre part, 
la taille du ménage a un effet négatif sur le revenu. Autrement dit, le bien-être du ménage diminue avec une augmentation du nombre d'individus en son sein. Les ménages dirigés par des femmes ainsi que ceux ayant un chef économiquement non occupé sont socialement plus vulnérables que ceux dirigés par des hommes et exerçant une activité économique respectivement.

Bien que n'étant pas significatifs, l'âge et l'âge au carré du chef de ménage ont un signe positif et négatif respectivement, ce qui veut dire que le revenu du ménage décrit une courbe en forme de $U$ renversé, c'est-à-dire qu'il croît progressivement durant toute la période d'activité, mais décroît progressivement vers l'âge de la retraite.

En plus d'être significatif, l'inverse du ratio de Mill (Lambda) a un signe positif, ce qui est de très bon augure, car cela signifie qu'il existe une corrélation positive entre, d'une part, le terme d'erreur de l'équation de (non) sélection et, d'autre part, le terme d'erreur de l'équation de revenu, ce qui permet de valider la méthode d'estimation utilisée.

\section{Impact des transferts de fonds sur la pauvreté}

Les transferts de fonds améliorent significativement le bien-être des bénéficiaires, car les gains de consommation qu'ils génèrent sont très largement positifs (Tableau 4). Ils permettent une baisse de $32,1 \%$ de l'incidence de la pauvreté qui passe de $40,8 \%$ à $27,7 \%$. Par ailleurs, la distance entre les pauvres et le seuil de pauvreté diminue très légèrement en présence des transferts. Ce qui signifie que les sommes reçues restent assez insuffisantes : le profil des migrants issus de ces ménages pourrait apporter davantage d'explications à un tel résultat. Bien que n'étant pas significatif, ce résultat pourrait tout de même se justifier par le fait que l'émigration internationale au Cameroun concerne en majorité les ménages aisés (71\%) ; ceux-ci bénéficiant en retour le plus de ses retombées financières comparativement aux pauvres. Les fonds reçus $\mathrm{n}$ 'entrainent pas un nivelage (une égalisation) des revenus parmi les pauvres. On observe au contraire une hausse des inégalités de revenu de 19,2 \% au sein de ce sous-groupe ; certains en bénéficiant plus que d'autres.

Tableau 4 : Impact des transferts de fonds sur la pauvreté

\begin{tabular}{|l|l|l|l|}
\hline \multicolumn{1}{|c|}{ Pauvreté } & \multicolumn{1}{c|}{ Sans transfert } & \multicolumn{1}{c|}{ Avec transferts } & \multicolumn{1}{c|}{ Écart (\%) } \\
\hline Dépense par tête & 550284 & 1779036 & $223,3^{* * *}$ \\
\hline Incidence & 40,8 & 27,7 & $-32,1^{* *}$ \\
\hline Profondeur & 11,3 & 10,6 & $-6,6$ \\
\hline Sévérité & 4,78 & 5,7 & 19,2 \\
\hline \multicolumn{2}{|l|}{$*^{* *} p<0,001}$.
\end{tabular}

Source : Données de I'IFORD, 2012.

Crédit : Eric Stève Tamo Mbouyou.

\section{Impact des transferts de fonds sur les inégalités de revenu}

En ce qui concerne l'impact des transferts de fonds sur les inégalités de revenu, on constate que leur présence tend à les renforcer (Tableau 5). Seuls les ménages non pauvres précisément ceux des $4 \mathrm{e}$ et $5 \mathrm{e}$ quintiles les plus 
riches en tirent un réel profit avec une amélioration de leur bien-être respectif quasi au-delà de $50 \%$. Pour le quintile riche, la migration se traduit donc par une hausse de la consommation moyenne. En d'autres termes, les ménages riches reçoivent plus de leurs émigrants que ce que ces derniers auraient gagné s'ils étaient restés travailler au Cameroun. Ce qui est loin d'être le cas chez les pauvres. Ce résultat se vérifie également au niveau de l'indice de Gini dont la valeur croît de 0,45 à 0,76 soit une hausse de $68,9 \%$ en présence des transferts, traduisant une exacerbation de ces inégalités.

Tout ceci confirme les précédents résultats où il est démontré, à travers la variation quasi nulle de la profondeur de la pauvreté, que les transferts de fonds au Cameroun sont surtout bénéfiques aux plus riches. Ces résultats sont similaires à ceux d'Adams et al. (2008) au Ghana et de Barham et Boucher (1998) au Nicaragua.

Tableau 5 : Impact des transferts de fonds sur les inégalités de revenu

\begin{tabular}{|l|l|l|l|}
\hline Quintile de bien-être & \multicolumn{1}{|c|}{ Sans transfert } & \multicolumn{1}{|c|}{ Avec transferts } & \multicolumn{1}{c|}{ Écart (\%) } \\
\hline Pauvre & 293551 & 166096 & $-43,4^{* * *}$ \\
\hline Non pauvre & 648607 & 2396758 & $269,5^{* * *}$ \\
\hline Q1 & 237374 & 114491 & $-51,8^{* * *}$ \\
\hline Q2 & 400878 & 245471 & $-38,8^{* * *}$ \\
\hline Q3 & 597779 & 409522 & $-31,5^{* *}$ \\
\hline Q4 & 558871 & 831995 & $48,9^{* * *}$ \\
\hline Q5 & 901232 & 8019190 & $789,8^{* * *}$ \\
\hline Indice de Gini & 0,45 & 0,76 & $68,9^{* * *}$ \\
\hline
\end{tabular}

Source : Données de l'IFORD, 2012.

Crédit : Eric Stève Tamo Mbouyou.

\section{Conclusion}

Cet article avait pour objectif d'évaluer la contribution des migrations internationales au développement des pays d'origine, à travers notamment la mesure de l'impact des transferts de fonds sur la pauvreté et les inégalités de revenu. À l'aide des données de l'enquête nationale sur l'impact des migrations Sud-Sud sur le développement au Cameroun réalisée en 2012 par I'IFORD, nous avons comparé la situation observée, c'est-à-dire en présence des transferts de fonds, à un scénario contrefactuel où le migrant resterait et travaillerait dans son pays d'origine.

Pour ce faire, nous avons recouru à la méthode des Moindres Carrés Ordinaires pour estimer le revenu des ménages non bénéficiaires puis nous avons utilisé les coefficients estimés pour prédire celui des ménages bénéficiaires en absence de migration ; le tout après avoir corrigé, à partir d'un mécanisme inspiré de celui de Barham et Boucher (1988), le biais endogénéité introduit par la sélectivité des ménages migrants. Cependant, l'approche utilisée comporte une limite à savoir qu'elle sous-tend implicitement l'impact sur le revenu actuel des fonds reçus par le ménage. Or un tel impact peut résulter des effets directs et/ou indirects des transferts antérieurs sur d'autres sources de revenus. 
II ressort des analyses que les transferts de fonds améliorent significativement le bien-être des bénéficiaires, car les gains de consommation qu'ils génèrent sont très largement positifs. Ils permettent une baisse de $32,1 \%$ de l'incidence de la pauvreté. Cependant, les sommes reçues demeurent assez insuffisantes étant donné que la distance moyenne qui sépare les pauvres du seuil de pauvreté reste quasiment la même que celle obtenue en absence de transferts.

Seuls les ménages les plus riches tirent un réel profit de l'émigration internationale, car les fonds reçus en retour de leurs émigrants sont bien au-dessus de ce que ces derniers auraient gagné s'ils étaient restés travailler au Cameroun. Ce qui est loin d'être le cas chez les pauvres. Cette situation est à l'origine d'une recrudescence des inégalités de revenu qui se traduit par une hausse de 68,9\% de l'indice de Gini.

Ces résultats qui sont somme toute prévisibles dans une certaine mesure nous livrent deux principaux enseignements pour l'action des pouvoirs publics. Premièrement, les migrations internationales à travers les envois de fonds qui en résultent participent concrètement au développement du pays d'origine, notamment à travers la lutte contre la pauvreté. Ceci suggère donc qu'une incitation des bénéficiaires pour une utilisation des fonds transférés à des fins productives plutôt que de consommation permettrait à terme de lutter encore plus efficacement contre cette pauvreté. Deuxièmement, malgré un effet positif tel que souligné précédemment, les envois de fonds des migrants sont tout de même à l'origine de dysfonctionnements sociaux caractérisés par une aggravation des inégalités de revenu au sein de la population : certaines couches sociales, en l'occurrence les plus favorisées, bénéficiant plus largement de ces fonds. Dans un tel contexte, une égalisation ou alors un nivelage de revenu entre ces différentes couches passe, entre autres, par des transferts monétaires des pouvoirs publics vers les ménages les plus pauvres. Toutefois, la soutenabilité d'une telle action dans le temps oblige par ailleurs que ces transferts sociaux soient couplés à des projets de développement et autres initiatives visant à réduire la dépendance de ces derniers à l'égard desdits transferts. 


\section{Références bibliographiques}

Adams Richard (2006) Remittances and Poverty in Ghana, World Bank Policy Research Working Paper 3838, 43 p.

Adams Richard (1991)The Economic uses and Impact of International Remittances in Rural Egypt, Economic Development and Cultural Change, 39 (4), pp. 695-722.

Adams Richard, Cuecuecha Alfredo and Page John (2008) The Impact of Remittances on Poverty and Inequality in Ghana, World Bank Policy Research Working Paper 4732, 41 p.

Adams Richard and Page John (2005) Do International Migration, Remittance Reduce Poverty in Developing Countries, World Development, 33 (10), pp. 1645-1669.

Adams Richard and Page John (2003) International Migration, Remittances and Poverty in Developing Countries, World Bank Policy Research Working Paper $3179,38 \mathrm{p}$.

Adepoju Aderanti (2011) Reflections on international migration and development in sub-Saharan Africa, African Population Studies, 25 (2), pp. 298-319.

Afaha John (2011) Migration, remittance and development in origin countries: evidence from Nigeria, African Population Studies, 27 (1), pp. 53-69.

Aggarwal Reena, Demirgüç-Kunt Asli and Peria Maria (2006) Do Workers' Remittances Promote Financial Development?, World Bank Policy Research Working Paper 3957, 40 p.

Anyanwu John and Erhijakpor Andrew (2010) Do International Remittances Affect Poverty in Africa, African Development Review, 22 (1), pp. 51-91.

Barham Bradford and Boucher Stephen (1998) Migration, remittances, and inequality: estimating the net effects of migration on income distribution, Journal of Development Economics, 55, pp. 307-331.

Boutin Delphine (2011) Envoi de fonds et allocation du temps des enfants au Niger : I'effet indirect des chocs négatifs, LAREFI Working Paper CR11-EFI/05, Bordeaux, Université Bordeaux IV, 33 p.

Chami Ralph, Barajas Adolfo, Cosimano Thomas, Fullenkamp Connel, Gapen Michael and Montiel Peter (2008) Macroeconomic Consequences of Remittances, IMF Occasional Paper 259, 94 p.

Delaunay Daniel (2006) Relations entre la pauvreté, la migration et les mobilités : dimensions territorial et contextuelle, Reunión de Expertos sobre Población y Pobreza en América Latina y el Caribe (14 y 15 de Noviembre 2006, Santiago, Chile), 33 p.

Development Research Centre on Migration, Globalisation and Poverty (2007) Global Migrant Origin Database, University of Sussex, [online]. URL: http://www. migrationdrc.org/research/typesofmigration/global_migrant_origin_database. html

Efionayi-Mäder Denise, Perroulaz Gérard et Schümperli Catherine (2008) Migration et développement : les enjeux d'une relation controversée, Annuaire suisse de politique de développement, 27 (2), pp. 11-20.

Frank Reann and Hummer Robert (2002) The Other Side of the Paradox:The Risk of Low Birth Weight among Infants of Migrant and Non migrant Households within Mexico, International Migration Review, 36 (3), pp. 746-765. 
Gubert Flore (2005) L'impact des transferts de fonds sur le développement des pays d'origine : le cas de l'Afrique, in OCDE Éd., Migrations, transfert de fonds et développement, Paris, Éditions OCDE, pp. 43-63.

Gubert Flore, Lassourd Thomas et Mesplé-Somps Sandrine (2010) Transferts de fonds des migrants, pauvreté et inégalités au Mali. Analyse à partir de trois scénarii contrefactuels, Revue économique, 6 (10), pp. 1023-1050.

Guilmoto Christophe et Sandron Frédéric (2003) Migration et développement, Paris, La Documentation Française, 142 p.

Institut National de la Statistique (2008) Enquête Camerounaise auprès des Ménages, Rapport principal, 190 p.

Kamdem Pierre (2007) Camerounais en Île-de-France : Dynamiques migratoires et stratégies d'intégration socio-spatiale différenciées, Paris, L'Harmattan, 313 p.

Khachani Mohamed (2004) Les déterminants de l'émigration internationale au Maghreb, in AIDELF Éd., Les migrations internationales : observation, analyse et perspectives (Actes du colloque de Budapest, 2004), AIDELF, pp. 209-222.

Lachaud Jean-Pierre (2005) Crise ivoirienne, envois de fonds et pauvreté au Burkina Faso, Tiers-Monde, 46 (183), pp. 651-673.

Lachaud Jean-Pierre (1999) Envois de fonds, inégalités et pauvreté au Burkina Faso, Tiers-Monde, 40 (160), pp. 793-827.

López-Cordova Ernesto (2006) Globalization, Migration and Development: The Role of Mexican Migrant Remittances, INTAL-ITD Working Paper 20, 60 p.

Mimche Honoré (2009) Émigration internationale et développement local au Cameroun, Actes des JERSIC, 23 p.

Ratha Dilip, Mohapatra et Silwal Ani (2011) Le Recueil de statistiques 2011 sur les migrations et les envois de fonds, 2e édition, Banque mondiale, 275 p. [en ligne] consulté le 15/01/2012. URL : http://siteresources.worldbank.org/INTPROSPECTS/ Resources/334934-1110315015165/Factbook2011French.pdf

Schmelz Andrea (2007) La diaspora camerounaise en Allemagne : sa contribution au développement du Cameroun, Eschborn, GTZ, 30 p.

Taylor Edward, Mora Jorge, Adams Richard and López-Feldman Alejandro (2008) Remittances, Inequality and Poverty: Evidence from Rural Mexico, in Migration and Development within and across Borders: Research and Policy Perspectives on Internal and International Migration, Geneva, IOM, pp. 101-128.

Wanner Philippe (2008) L'apport des migrants au développement : une perspective économique, Annuaire suisse de politique de développement, 27 (2), pp. 121-131.

Yang Dean (2008) International Migration, Remittances and Household Investment Evidence from Philippine Migrants' Exchange Rate Shocks, Economic Journal, 118 (528), pp. 591-630.

Zourkaléini Younoussi, Mimche Honoré, Nganawara Didier, Nouetagni Samuel, Seke Kouassi, Kouam Choupi Nadège, Hamadou Saidou et Ledoux Tjomb JeanPierre (2013) Un regard vers le Sud: Profil des migrants et impact des migrations sur le développement humain au Cameroun, Rapport de Recherche, Genève, OIM, $182 \mathrm{p}$. 


\section{Annexe}

Tableau 6 : Probabilité d'une non-participation du ménage à l'émigration internationale

\begin{tabular}{|c|c|c|}
\hline Variables & Coefficients & $\begin{array}{c}\text { Effets } \\
\text { marginaux }\end{array}$ \\
\hline \multicolumn{3}{|l|}{ Milieu de résidence } \\
\hline Yaoundé/Douala & Réf. & Réf. \\
\hline Urbain & $-0,0643$ & $-0,0244$ \\
\hline Rural & $-0,0278$ & $-0,0105$ \\
\hline Score d'actifs possédés (Log) & $-0,3167 * * *$ & $-0,1197 * * *$ \\
\hline Taille du ménage (Log) & 0,1054 & 0,0398 \\
\hline Nombre d'instruits (Log) & $-0,2458 * *$ & $-0,0929 * *$ \\
\hline Sexe du chef de ménage ( $0=$ Homme, $1=$ Femme $)$ & $-0,2782 * *$ & $-0,1073 * *$ \\
\hline Âge du chef de ménage & $-0,0175$ & $-0,0066$ \\
\hline Âge du chef de ménage au carré & 0,0000 & 0,0000 \\
\hline Situation d'activité du CM ( 0 = Non occupé, 1 = Occupé) & 0,0773 & 0,0294 \\
\hline \multicolumn{3}{|l|}{ Langue parlée } \\
\hline Français uniquement & Réf. & Réf. \\
\hline Anglais uniquement & $-0,4777^{*}$ & $-0,1868 * *$ \\
\hline Français et anglais & $-0,2358 * * *$ & $-0,0902^{*}$ \\
\hline Constante & 1,7293 & \\
\hline
\end{tabular}




\section{Eric Stève Tamo Mbouyou}

\section{Envois de fonds des migrants, pauvreté et inégalités de revenu au Cameroun}

Cet article mesure l'impact des envois de fonds des migrants à destination du Cameroun sur la pauvreté et les inégalités de revenu, à l'aide des données de I'enquête nationale sur "L'impact des migrations Sud-Sud sur le développement au Cameroun " réalisée en 2012 par I'Institut de Formation et de Recherche Démographiques (IFORD). En considérant les envois de fonds comme étant des substituts potentiels des gains produits localement par le ménage en absence de migration, il ressort des analyses présentées que ces fonds améliorent significativement le bien-être des bénéficiaires, grâce notamment aux gains de consommation qu'ils génèrent et qu'ils permettent une réduction de l'incidence de la pauvreté. Cependant, comme seuls les ménages les plus aisés en bénéficient le plus, cela a pour conséquence un renforcement des inégalités.

\section{..: Remittances, Poverty and Income Inequality in Cameroon}

This paper measures the impact of remittances from migrants to Cameroon on poverty and income inequality using data from the national survey on "The Impact of South-South Migration on Development in Cameroon" conducted in 2012 by the Institute of Training and Demographic Research. Considering remittances as potential substitutes gains generated locally by the household in the absence of migration, it analyzes showed that these funds significantly improve the well-being of beneficiaries and reduce the incidence of poverty. However, only the wealthiest households benefit most. This has resulted in greater inequality.

\section{... Las remesas de los emigrantes, la pobreza y la desigualdad de ingresos en Camerún}

En este trabajo se mide el impacto de las remesas de los migrantes a Camerún en los datos de pobreza y desigualdad de ingresos usando de la encuesta nacional sobre «El impacto de la migración Sur-Sur para el Desarrollo en Camerún", realizado en 2012 por el Instituto de Formación e Investigación Demográfica. Teniendo en cuenta las remesas como sustitutos potenciales ganancias generadas localmente por la casa en la ausencia de la migración, que los análisis mostró que estos fondos a mejorar significativamente el bienestar de los beneficiarios y reducir la incidencia de la pobreza. Sin embargo, sólo los hogares más ricos se benefician más. Esto ha resultado en una mayor desigualdad. 